\title{
L'allemand comme langue imaginaire chez Victor Hugo et Balzac
}

Jeanne Bem

Université de la Sarre

Les rapports entre la France et l'Allemagne sont toujours d'une brûlante actualité. Il est question ici de retourner à l'avant-dernier siècle et essentiellement au moment romantique. Il s'agit de saisir quelque chose de ces rapports entre la France et l'Allemagne chez deux écrivains français du premier XIXème siècle : Hugo et Balzac, en analysant des textes, des effets textuels, sur quelques exemples précis.

Qui dit rapport entre peuples, dit: définition de l'identité nationale, et dit forcément: langue. L'identité se définit dans l'opposition à l'autre, et si la langue d'un peuple est une autre langue que celle du peuple voisin, cela renforce nécessairement son identité. À l'inverse, l'autre attire, précisément à cause de sa langue et de sa culture autres. Les rapports franco-allemands ont toujours été marqués par cette ambivalence. La génération romantique a été influencée par le livre de Mme de Staël, De l'Allemagne (conçu à partir de 1804 et publié en 1814) : c'est un livre d'envergure européenne, qui se propose en médiateur entre l'esprit français et le génie allemand. Pour ces écrivains des années 1830-1840, la langue allemande n'est donc pas juste un vecteur de communication, c'est la langue des philosophes et des poètes allemands : elle est alors fortement chargée de valeurs positives, c'est une langue que l'on peut avoir envie de s'approprier. En même temps, le génie allemand reste irréductiblement autre, étrange. Quant à l'Allemagne, c'est un espace attirant, un espace à explorer, mais aux frontières mouvantes, à la définition incertaine.

Il ne faut pas oublier que la langue fonde aussi l'identité de chaque individu, en tant qu'elle est sa langue «maternelle ». Je me réfère au livre de John E. Jackson, Passions du sujet. Essais sur les rapports entre psychanalyse et littérature (1990) : il souligne que la mère, en même temps qu'elle apporte à l'enfant nourriture et affection, l'initie à un rapport joueur, euphorique, avec la matière sonore des mots. Deviendrait écrivain l'enfant qui (selon des modalités évidemment variables, car chacun a son histoire propre) aurait été amené, dans sa relation avec sa mère, à surévaluer la langue, à accorder à la langue, au jeu avec la langue, une valeur supérieure à toute autre activité1.

Les textes que nous allons analyser se trouvent donc au croisement de l'individuel et du collectif. On voit mieux pourquoi, plutôt que d'une langue réelle, on peut parler

\footnotetext{
${ }^{1}$ J'en resterai à cette conception générale et je n'essaierai pas de «psychanalyser » Victor Hugo ou Balzac.
} 
d'une langue imaginaire. Chacun de ces écrivains est fasciné par la langue allemande, il la voit comme une langue qui pourrait contaminer sa langue maternelle, ou même s'y substituer (dans l'imaginaire bien sûr). Le rapport de ces écrivains à l'allemand s'exprime parfois dans des commentaires, attribués au narrateur ou aux personnages. En tout cas il y a une conscience de la langue, de l'altérité des langues, au cœur du texte. Le plus intéressant, ce sont les citations en allemand, c'est le travail de l'allemand sur le français et le jeu inter-linguistique qui en résulte. C'est ici que peuvent apparaître les processus inconscients à l'œuvre dans les textes. Le phénomène inter-linguistique vient inscrire dans le texte littéraire des figures invisibles. Si l'on arrive à les décrypter, on aperçoit quelque chose de l'expérience intime du sujet, de ses attentes vis-à-vis de la langue et de l'écriture, de la capacité de l'écrivain à créer "de la langue », ou à créer " du texte » avec de la langue, de son rêve ou de sa peur de passer "de l'autre côté » de la barrière des langues, ou encore - chose plus difficile à concevoir - de passer de l'autre côté de la langue. Dans une non-langue ? Dans l'outre-langue ? Dans un sans-langue ? Dans un néant?

On connaît les incertitudes de l'espace allemand. "L'Allemagne », « de l'Allemagne »: on a une entité englobante, la marque aussi d'une provenance, d'une origine. Mais cet espace, cette histoire restent une énigme. Dans Le Rhin (1842), quand Victor Hugo arrive aux pages de sa Conclusion, il retourne deux siècles en arrière, au début du XVII ${ }^{\text {ème }}$ siècle, pour rappeler ce qu'était alors le Saint Empire romain germanique : quatre-vingt-dix-huit états - des royaumes, des archiduchés, des duchés, des principautés, des comtés, des margraviats, des évêchés, des villes libres, avec des statuts et des modes de fonctionnement différents (Hugo CEuvres 370)! Il n'y a donc pas «l'Allemagne », mais « des » Allemagnes. La boussole n'est pas toujours sûre non plus : l'Allemagne se situe généralement au nord, ce qui lui amalgame parfois les Flandres et les Pays-Bas; mais quelqu'un comme Nerval a tendance à la situer à l'est, et même au sud-est: assimilant Constance à Constantinople, il assimile son expérience allemande (un hiver passé à Vienne, surtout) à sa quête de l'Orient (Huré 31-33).

Si l'on a du mal à apercevoir les confins de cet espace, qui varient au gré des guerres et des traités, il y a un axe : le Rhin. Victor Hugo en tout cas est fasciné par cet axe qui, selon lui, structure l'espace européen. C'est autour d'une remontée du Rhin, en commençant par la Champagne, la Lorraine, la Belgique wallonne, en passant ensuite par Aix-la-Chapelle, Cologne, Coblence, Mayence, en continuant vers Strasbourg et la Suisse, qu'il organise son récit de voyage. Le Rhin n'est pas un vrai voyage, c'est le récit ou le roman d'un voyage présenté sous forme de lettres et qui regroupe trois vrais voyages partiels et dispersés, effectués en 1838, 1839 et 1840. C'est un livre fondé de surcroît sur le plagiat de plusieurs ouvrages documentaires et guides touristiques. Quand Le Rhin est publié, on est en pleine crise franco- 
allemande à propos de la rive gauche du Rhin (Savy). Victor Hugo lui-même ne manque pas de faire, dans la quatorzième lettre, une longue digression historique, il remonte au Rhin romain, il rappelle le deuxième partage de l'empire de Charlemagne en 855, l'attribution de la Lotharingie à Lothaire (c'est là-dessus que se fonde la revendication de la rive gauche par la France), et il souligne que par trois fois dans l'Histoire, sous Pépin-le-Bref, sous Charlemagne, sous Napoléon, la France a même possédé la rive droite du Rhin (Hugo CEuvres 108)²! Parlant de lui-même à la troisième personne dans sa Préface, Victor Hugo écrit : «L'Allemagne, il ne le cache pas, est une des terres qu'il aime et une des nations qu'il admire. Il a presque un sentiment filial pour cette noble et sainte patrie de tous les penseurs. S'il n'était pas Français, il voudrait être Allemand. »(Hugo CEuvres 9) Naturellement notre étude du texte va nuancer cette profession de foi.

Victor Hugo a certaines tendances polyglottes, mais bizarrement il évite plutôt l'allemand. (Des souvenirs d'enfance le poussent vers l'espagnol ; en exil il vit aux confins de l'anglais; et il n'oublie jamais le latin, sa langue étrangère de prédilection, encore une langue de l'enfance : on est étonné du nombre d'inscriptions latines et de citations en latin qui jalonnent Le Rhin; on pourrait presque penser que le latin de la Romania - cette aire linguistique marquée par l'influence romaine et qui englobe l'axe du Rhin - supplante pour lui la langue vernaculaire germanique.) En fait il connaît mal l'allemand et ne semble pas avide d'améliorer ses connaissances. Cela va de pair avec un certain désintérêt pour l'état présent des pays traversés - Victor Hugo est plus « antiquaire » (archéologue, érudit du passé) que journaliste. Je cite la notice de Evelyn Blewer dans l'édition Bouquins : «Il semble croire - l'ignorance de la langue aidant - que le Rhin allemand est profondément napoléonien, et que l'état d'esprit est celui d'une pax franca hypothétique d'avant le traité de Vienne. » (Hugo CEuvres 1236)

Ce cadre posé, voyons la trente-septième lettre (Hugo CEuvres 349-351). Le voyageur romantique approche de la fin de son parcours quand il arrive à Schaffhouse, une ville pittoresque qu'il évoque au début de la lettre "perchée dans la montagne, coupée par le Rhin qui se tord dans son lit de roches avec une grande clameur». Le début de la lettre est typique des manies du voyageur. Plus qu'aux choses, il s'intéresse aux mots - voyez sa digression qui porte d'abord sur la forme phonétique du nom de la ville, puis sur l'étymologie :

«Platine écrit Schaphuse, Strumphius écrit Schapfuse, Georges Bruin écrit Shaphusia, et Miconnis écrit Probatopolis. Tirez-vous de là. » (Hugo CEuvres 349)

Il prétend se moquer des « antiquaires » mais lui-même ne fait pas autre chose. Nous sommes en Suisse alémanique - autre façon de revivre la plasticité de l'espace

\footnotetext{
${ }^{2}$ Je donne ces informations seulement pour faire comprendre à quel point les repères géographiques, historiques, politiques sont fluctuants, à quel point l'identité française, dans ce type de discours, est près de fusionner avec l'identité allemande.
} 
appelé «Allemagne ». Puis, de mots allemands, il n'y en a guère, ou alors ils sont bizarres, déformés ; leur sens pose énigme : dans Schaffhausen, doit-on identifier une barque ou un mouton? Et comme d'habitude, c'est encore le latin qui fournit le repère stable, indiscutable, le repos intellectuel qu'apporte, quand on a lu le recto, le verso attendu de la page :

«[...] j'ai vu au-dessus de l'ogive cette inscription: SALVS EXEVNTIBVS. J'en ai conclu qu'il y avait probablement de l'autre côté : PAX INTRANTIBVS. J'aime cette façon hospitalière. » (Hugo CEuvres 349)

Le voyageur a besoin d'être rassuré, il va jusqu'à nous produire, comme si elle était là sous nos yeux, une deuxième inscription latine qu'il vient d'inventer dans sa tête. Et bien entendu le latin ne trompe jamais, c'est la langue utopique, dans sa transparence et son universalité. Une langue morte aussi, qui n'a plus qu'une demivie. La langue des inscriptions dans la pierre... Il y aurait à dire sur le latin de Victor Hugo et sur son bonheur à communiquer dans la belle langue défunte de l'Occident chrétien, mais ce serait une autre étude.

Toute la tonalité du début du texte est ludique et nous prépare à l'anecdote de la «Calaïsche à la choute ». À Schaffhouse, le voyageur se rend à l'auberge :

«On m'a apporté un dîner français, servi par un garçon français, avec une carte en français. Quelques originalités, sans doute involontaires, se mêlaient, non sans grâce, à l'orthographe de cette carte. Comme mes yeux erraient parmi ces riches fantaisies du rédacteur local, cherchant à compléter mon dîner, au-dessous de ces trois lignes :

Haumelette au chantpinnions,

Biffeteque au craison,

Hépole d'agnot au laidgume,

je suis tombé sur ceci :

Calaïsche à la choute, - 10 francs.

Pardieu ! me suis-je dit, voilà un mets du pays : calaïsche à la choute. Il faut que j'en goûte. Dix francs! cela doit être quelque raffinement propre à la cuisine de Schaffhouse. J'appelle le garçon. - Monsieur, une calaïsche à la choute. Ici le dialogue s'engage en français. Je vous ai dit que le garçon parlait français.

- Vort pien, monsir. Temain matin.

- Non, dis-je, tout de suite.

- Mais, monsir, il est pien tard.

- Qu'est-ce que cela fait?

- Mais il sera nuit tans eine hère.

- Eh bien?

- Mais monsir ne bourra bas foir.

- Voir ! voir quoi ? Je ne demande pas à voir.

- Che ne gombrends bas monsir.

- Ah çà ! c'est donc bien beau à regarder, votre calaïsche à la choute ?

- Vort peau, monsir, atmiraple, manifigue!

- Eh bien, vous m'allumerez quatre chandelles tout autour.

- Guadre jantelles ! Monsir choue. (Lisez : Monsieur joue.) Che ne gombrends bas.

- Pardieu ! ai-je repris avec quelque impatience, je me comprends bien, moi, j'ai faim. Je veux manger. 
- Mancher gouoi ?

- Manger votre calaïsche.

- Notre calaïsche?

- Votre choute.

- Notre choute! Mancher notre choute! Monsir choue. Mancher la choute ti Rhin!

Ici je suis parti d'un éclat de rire. Le pauvre diable de garçon ne comprenait plus, et moi, je venais de comprendre. J'avais été le jouet d'une hallucination produite sur mon cerveau par l'orthographe éblouissante de l'aubergiste. Calaïsche à la choute signifiait calèche à la chute." (Hugo CEuvres 350)

On peut lire cette page comme un morceau d'humour - en se souvenant que l'humour noir n'est jamais loin. Qu'est-ce qu'il y a de légèrement inquiétant dans cette histoire ? Le dialogue est en quelque sorte bilingue, car le garçon ne parle certes pas la même langue que le voyageur : «Ici le dialogue s'engage en français » est évidemment ironique. Le malentendu est de type classique, la surdité de chacun des deux interlocuteurs est fondée justement sur la langue, sur le fait que des mots français ont été altérés. À quoi s'ajoute que les mots calaïsche à la choute ont été écrits sur le menu, où ils ne devraient pas se trouver. La tournure «à la » les a rattachés à un paradigme erroné. Ce déplacement contribue à déplacer leur sens. De fait, le texte du menu est très inventif (l'haumelette n'est pas très loin de l'hommelette de Lacan). Mais le lecteur qui réfléchit un peu voit bien que la rédaction du menu doit plus à Victor Hugo et à son goût des calembours qu'à ses supposés protagonistes de langue alémanique.

Dans le dialogue qui s'ensuit, les répliques du garçon brouillent la piste, car il parle effectivement un français germanisé. Mais il le fait selon un code des transformations phonétiques qui a une vieille tradition dans la littérature et au théâtre, quand il s'agit de représenter quelqu'un qui parle avec l'accent allemand. Cette représentation n'est pas réaliste, elle est de pure convention. Cela consiste à remplacer systématiquement les sourdes par des sonores et les sonores par des sourdes : voyez le jeu entre $/ \mathrm{t} / \mathrm{et}$ /d/ ou entre /p/ et /b/ d de même, le /j/ devient /ch/ et inversement le /ch/ devient /j/. Donc ce n'est pas que le locuteur affecté de l'accent étranger soit incapable de prononcer certains sons, c'est qu'un code (à visée humoristique) veut que l'Allemand emploie toujours les sons à contretemps.

Mais il est à noter que la communication passe. Le rythme question-réponse ne faiblit pas. Les déformations qui obstruent les répliques du garçon sont corrigées dans les répliques du voyageur. Le seul nœud de résistance est la calaïsche à la choute. Déplacé dans un paradigme qui n'est pas le sien, ce syntagme échappe de surcroît aux transformations phonétiques habituelles: sinon nous aurions par exemple joude ou jude, et pas choute. (On voit à quel point tout ceci est fabriqué et comment le lecteur est manipulé.) Leçon : il suffit de changer très peu de chose à un mot qui nous est familier (dans notre cas : mettre une graphie allemande pour le son /ch/ à calaïsche ainsi qu'une une diphtongue inattendue, remplacer une voyelle par une 
autre dans choute) - et aussitôt nous ne reconnaissons plus rien, le mot devient pour nous totalement opaque. Autre leçon : il fallait cette longue remontée du livre et du Rhin, jusqu'à cette fameuse chute assourdissante de Schaffhouse, pour faire «entendre» au lecteur français sa propre langue comme autre, opaque, étrangère. Pour lui donner le petit frisson de la perte de sa langue.

Hugo est poète, il le prouve en portant une grande attention à l'écrit. Tout le texte est un jeu fondé sur la non-coïncidence entre l'oral et l'écrit, poussée jusqu'aux limites les plus folles, à partir du moment où par exemple une sourde remplace comme par inadvertance une sonore. On surprend le poète en train de «laisser l'initiative aux mots », ce qui l'amène à créer dans les répliques du garçon des monstres et des réalités impossibles. La beauté devient une peau, une exclamation se transforme en figue, il surgit de mystérieuses jantelles, et nous sommes invités à manger retournant ainsi, grâce à l'imaginaire des mots, à un stade pulsionnel infantile : monsieur joue - une calèche entière assaisonnée d'une chute d'eau! D'une chute d'eau : que dis-je ? de la cataracte du Rhin! En fait le lecteur est invité à " avaler » dans une seule gorgée gargantuesque tout le voyage (la calèche) et tout le livre (Le Rhin) qu'il vient de lire. Dommage que l'explication finale aplatisse l'effet: il n'en reste pas moins que Victor Hugo a parfois anticipé sur le surréalisme et sur l'absurde.

Mais justement, il s'est peut-être amusé à se faire peur. Défigurer sa langue maternelle est une transgression passagère, un petit meurtre symbolique qu'on n'accomplit qu'en passant, comme en rêve, pour avoir croisé cette autre langue, l'allemand. L'allemand de Schaffhouse (il faut le dire) est un allemand fantasmé, un allemand fantastique, un fantôme d'allemand. Cette langue n'existe qu'en creux dans ce texte, elle ne s'actualise que par l'action déformante qu'elle exerce sur son autre langue, le français. Cette action ressemble assez à l'action exercée par la censure sur le contenu manifeste des rêves (comme Freud s'en explique dans la Traumdeutung). Si la pulsion hugolienne décelable dans ce texte est de jouer avec sa langue, de retourner pour un instant en-deçà de la langue, dans les limbes d'avant l'enfance même, on peut dire que l'allemand lui apporte la force de compression, de destruction. Les relations avec l'autre ont toujours une composante agressive. L'épisode révolutionnaire et napoléonien est encore proche. Cela contribue sans doute à faire que l'allemand ait cet effet sur le texte hugolien - bien que cette composante historico-politique soit gommée, puisque nous sommes en Suisse.

Quand on redevient sérieux et même un peu pompeux, on met l'humour de côté. En 1867, à l'occasion de l'Exposition universelle de Paris, l'éditeur d'une brochure intitulée Paris-Guide qui est destinée aux millions de visiteurs attendus, demande à Victor Hugo (pourtant éloigné, exilé à Guernesey) d'en écrire la préface (Hugo Écrits). Le poète saisit l'occasion, l'Européen visionnaire se réveille, il prévoit que la 
France de l'avenir rayonnera sur l'Europe future, il prophétise, emporté dans une grande envolée: «Unité de langue, unité de monnaie, unité de mètre, unité de méridien, unité de code [...] » (Hugo Écrits 235-236) ${ }^{3}$. C'est là un beau programme, et déjà en grande partie réalisé dans l'Union européenne aujourd'hui. Cependant, « unité de langue » demande qu'on s'y arrête. Ce n'est pas un enjeu du même ordre que les autres "unités », et cette fois les Européens auraient de quoi être inquiets: Victor Hugo n'a-t-il pas laissé échapper (à son insu) qu'il liquidait les langues européennes et que toute l'Europe allait parler français ? Ce serait grave, surtout si tout le monde se mettait à parler comme l'homme de Schaffhouse.

Passons à l'analyse des textes de Balzac. Je me suis intéressée au baron de Nucingen, un personnage étonnant qui apparaît dans plusieurs œuvres. Et d'abord, comment prononcer son nom ? à l'allemande? à la française? en combinant les deux prononciations (ce qui est d'usage encore aujourd'hui en Alsace) ? Écoutons le baron prononcer lui-même son propre nom (et n'en tirons pas de conclusion trop rapide) :

«Ui, mon envand, che suis le paron te Nichinguenne [...]» (Balzac Comédie vi 575)

C'est un personnage que le lecteur de Balzac a déjà aperçu fugitivement, dans Le Père Goriot (1834), c'est le mari de Delphine. Il est beaucoup plus présent dans trois œuvres qui se trouvent dans le tome VI de l'édition de la Pléiade. Il y a une nouvelle, La Maison Nucingen (1837), qui retrace l'histoire de l'ascension phénoménale de ce requin de la finance (Balzac emploie pour sa part l'image du loup-cervier, c'est-àdire du lynx) et qui raconte ses trois faillites, la troisième étant une faillite frauduleuse. Il y a un roman, César Birotteau (1838), qui a été composé par Balzac parallèlement à La Maison Nucingen : dans la nouvelle on trouve déjà des échos du roman, il y a des mentions de la malheureuse histoire du parfumeur Birotteau et de sa faillite (une faillite honnête, contrairement à la faillite de Nucingen). Le personnel romanesque des deux œuvres est souvent le même. Enfin, le baron va jouer un rôle secondaire dans un roman plus tardif, Splendeurs et misères des courtisanes (1845), dont l'histoire se situe dans le sillage des Illusions perdues. Le baron a maintenant soixante ans et il est le protecteur berné de la courtisane Esther.

Pourquoi se concentrer sur Nucingen? C'est parce que ce personnage est un cas. Certes, écrit Pierre Citron dans son Introduction à La Maison Nucingen dans la Pléiade, ce financier inventé par Balzac a des modèles dans le réel (tels que les Rothschild, ou encore Fould, ou bien Ouvrard), certes il est représentatif du capitalisme au milieu du XIXème siècle, mais son traitement romanesque le met à part des autres personnages balzaciens. "Nucingen [...] n'est qu'une silhouette (il est gras), un nom, et moins qu'une voix - un accent: quelque chose de quasi impalpable.»(Balzac Comédie vi 324). Dans les affaires, poursuit le critique, le

\footnotetext{
${ }^{3}$ Le mot « code » désigne ici l'espace juridique.
} 
banquier Nucingen « ne manie que des signes dont la valeur est conventionnelle et parfois fictive », il représente la richesse « algébrique ».

On aura compris que Nucingen est un personnage qui se résume à ses émissions de parole. On remarque que Pierre Citron ne parle pas de son discours, ni de sa langue, il dit juste : « un accent ». Cet accent, l'accent allemand pour dire vite, nous venons de le rencontrer avec ses conventions littéraires, dans le texte de Victor Hugo. Mais chez Balzac les choses se compliquent. D'abord, il ne s'agit pas cette fois de récits de voyage. On ne va pas à la rencontre de l'autre - comme peuvent le faire de grands voyageurs romantiques, Hugo, Stendhal, Alexandre Dumas, Nerval, ou Flaubert... Ici c'est l'autre qui en quelque sorte s'introduit dans la place. C'est « l'Allemagne », cet espace étrange, qui vient à nous, sous la forme de «l'accent». La question de l'origine est posée dans les trois romans de Balzac, avec insistance et avec quelque incohérence. Le lecteur est confronté à trois formulations différentes : Nucingen est 1. Alsacien, fils de juif converti. 2. Juif allemand. 3. Juif polonais ${ }^{4}$. Résignons-nous au fait que Balzac véhicule l'antisémitisme ambiant. Mais il le fait sans animosité particulière vis-à-vis du personnage. Dire que Nucingen est converti évacue le problème religieux. Ce qui ressort surtout, c'est que le banquier est vraiment «l'autre ». Alsacien, Allemand et Polonais sont des définitions quasiment équivalentes dans l'esprit de Balzac et Juif les condense comme une sorte de mot-clef magique. D'où vient Nucingen? De là d'où viennent apparemment tous les banquiers (et tous les Juifs): de Strasbourg, d'Alsace, de ce lieu déjà vague en lui-même et indéfinissable (y parle-t-on seulement français ? on peut en douter ${ }^{5}$ ) derrière lequel il y a, quelque part au-delà du Rhin, une entité encore plus vague, l'Outre-Rhin en somme ! Les banquiers, juifs ou non, arrivent de là et malgré leur « baragouin $»^{6}$, ils conquièrent la «place » de Paris (le mot est à la fois militaire et boursier). Nucingen est puissant et recherché. Les autres protagonistes le craignent et le respectent. Ils supportent patiemment l'accent. Le baron n'est pas complètement négatif d'ailleurs. Il est vrai qu'il mène en bateau le pauvre César Birotteau. Mais il favorise Rastignac, l'ex-amant de sa femme.

Cet «accent» pourrait être comme la trace laissée dans le texte romanesque par le redoutable et opaque fonctionnement de l'argent. Balzac prend parfois l'argent pour

\footnotetext{
${ }^{4}$ Voici des textes à l'appui : «Ouvrard est le seul qui ait deviné que cet Alsacien, fils de quelque juif converti par ambition [...]» (Balzac La Comédie humaine vi 338) ; «Le fin baron, pour avoir des motifs de revenir sur des paroles bien données et mal entendues, avait gardé l'horrible prononciation des juifs allemands qui se flattent de parler français » (Balzac Comédie vi 232); «[...] elle n'écoutait pas du tout le baron [...] lui parlant dans son patois de juif polonais [...] 》 (Balzac Comédie vi 645).

${ }^{5}$ Un témoignage parmi d'autres sur Strasbourg, celui de Nerval dans Lorely (1852), remontant à un voyage de 1838 : «Il faut bien l'avouer, on parle moins français à Strasbourg qu'à Francfort ou à Vienne, et de plus mauvais français, quand on le parle. Il est difficile de se faire comprendre des gens du peuple, et nous en sommes à nous demander ce qu'apprennent les enfants aux écoles mutuelles qu'on dit si fréquentées dans ce département. Peut-être savent-ils le latin. » (Nerval CEuvres iii 19).

${ }^{6}$ Même les Goncourt s'appliquent à transcrire l'accent de Rothschild : voir l'entrée du 9 mars 1862 (Goncourt Journal i 782).
} 
thème exclusif d'un roman - et singulièrement dans ces véritables romans financiers que sont César Birotteau et La Maison Nucingen. Bien qu'il se donne du mal pour démonter les mécanismes de sa circulation, on ne peut pas dire que tout soit limpide pour le lecteur. Ainsi l'opacité du discours du banquier correspondrait au voile qui recouvre les affaires financières pour le commun des mortels. Nucingen finit par acquérir ainsi une dimension mythique. Ce personnage immatériel qui n'existe que par ses paroles (qui seraient autant d'oracles ?) se distingue des autres personnages quand il participe aux dialogues, en ce que ses répliques à lui sont toujours imprimées en italiques. Il est le personnage qui, quand il ouvre la bouche, fait aussitôt surgir les italiques sur la page ${ }^{7}$. En général, Balzac réserve les italiques : aux locutions, aux mots de métier, d'argot, aux expressions favorites d'un personnage, aux titres et affiches, aux citations, aux langues étrangères. Nucingen, comme Kolb, l'Alsacien représenté dans Illusions perdues (Balzac Comédie v), est supposé parler en français. Est-ce à dire que ce français-là est une langue étrangère ? Balzac suggère une catégorie intermédiaire : des "paroles semi-françaises » (Balzac Comédie vi 232). Ou devrait-on plutôt, dans un souci de réalisme, ranger le discours opaque de Nucingen dans le paradigme des ruses de métier ? Le rapprochement a été fait entre le baragouin de Nucingen et le bégaiement du père Grandet (TerrasseRiou 121 et suiv.). Il faut noter que c'est après avoir été lui-même berné par un Juif qui affectait d'être sourd, que le vigneron a mis au point sa méthode (Terrasse-Riou 124) : c'est du moins ce que nous raconte complaisamment le narrateur dans Eugénie Grandet. «L'accent » de Nucingen se situerait entre sociolecte et idiolecte.

Mais on sent bien que dans son cas il s'agit d'autre chose, que ce n'est pas juste un banquier qui mène ses affaires louches à l'abri d'un discours volontairement rendu incompréhensible. Bien sûr, on le voit embobiner le parfumeur Birotteau : c'est un exemple qui pourrait suggérer une fonctionnalité du discours du baron. Il est bon peut-être de prendre connaissance des paroles qu'il susurre à Birotteau :

"Ti Dilet breind lei plis fiffoe eindéred à vus », dit-il.

« Enfin, pensa le pauvre négociant, nous arrivons à la question. »

"Afec sa leddre, vis affez tan mâ mésson eine grétid ki n'ed limidé ké bar lais pornes te ma brobre vorteine... » (Balzac Comédie vi 232)

[je traduis :

« Du Tillet prend le plus vif intérêt à vous. »

«Avec sa lettre, vous avez dans ma maison [ma banque] un crédit qui n'est limité que par les bornes de ma propre fortune. »]

\footnotetext{
${ }^{7}$ «L'italique fut inventé par les Alde, à Venise : de là son nom », c'est ce que David explique à Ève dans Illusions perdues quand il lui fait l'histoire du papier (Balzac Comédie v 219). Il est à noter qu'un Alsacien apparaît dans ce roman : c'est Kolb, le serviteur dévoué du jeune inventeur d'Angoulême. Kolb est très actif dans la troisième partie. Bien entendu il est caractérisé par son accent. Mais pour ce personnage du peuple, simple et bon, les transcriptions restent dans des limites décentes, le lecteur reconnaît le code habituel. "Si matame feut addentre... » (Balzac Euvres 620). Les paroles de Kolb sont, comme celles de Nucingen, toujours rendues en italiques.
} 
Aidé des commentaires du narrateur, rendu donc sensible à l'hyperbole des promesses du banquier, le lecteur n'est pas dupe, quoique le parfumeur le soit :

Le baume exhilarant que contenait l'eau présentée par l'ange à Agar dans le désert devait ressembler à la rosée que répandirent dans les veines du parfumeur ces paroles semifrançaises. Le fin baron, pour avoir des motifs de revenir sur des paroles bien données et mal entendues, avait gardé l'horrible prononciation des juifs allemands qui se flattent de parler français. (Balzac Comédie vi 232)

Cependant il faut constater une contradiction dans ce commentaire: "mal entendues", les paroles de Nucingen? Dans le cas des discours de Nucingen, exactement comme dans le dialogue à Schaffhouse imaginé par Victor Hugo, la communication entre les personnages n'est jamais perturbée. Le dialogue est fluide. Nucingen baragouine, les autres lui répondent imperturbablement, comme si de rien n'était. En voici des exemples :

«Savez-vous, baron, lui dit de Marsay, que vous avez maigri considérablement ? et l'on vous soupçonne de violer les lois de la nature financière.

- Chamais! dit le baron.

- Mais si, répliqua de Marsay. On ose prétendre que vous êtes amoureux.

- C'esde frai, répondit piteusement Nucingen. Chai zoubire abbrest kèque chose t'ingonni. [...]

- Où l'avez-vous rencontrée, cette jeune innocente ? demanda Rastignac.

- An foidire, hâ minouid, au pois de Finzennes » (Balzac Comédie vi 497).

«Esder, dit-il en lui lâchant la main, et la repoussant avec un léger mouvement d'humeur, fus ne m'égoudez bas!

- Baron, tenez, vous baragouinez l'amour comme vous baragouinez le français.» (Balzac Comédie vi 645)

On peut remarquer que dans le troisième roman, Splendeurs et misères, Nucingen finit par perdre de son aura. Endossant le rôle du vieillard amoureux, qui est un rôle de comédie, il se laisse manipuler par une prostituée et un bandit (Esther et Vautrin). Les autres personnages se moquent de lui. De Marsay et Rastignac sont clairement ironiques. "Cette jeune innocente", c'est Esther! Dans Splendeurs et misères, un parallèle est fait entre la finance véreuse et l'amour vénal. "Baron, tenez, vous baragouinez l'amour comme vous baragouinez le français. » Le reproche est injuste. Le baragouin de Nucingen emblématise deux types de fraude : la fraude dans les affaires et la fraude dans la communication en affaires. Mais il est sincère en amour et son baragouin se retourne contre lui.

Parmi les abondants commentaires du narrateur, retenons celui-ci : « [...] son patois de juif polonais, dont les singulières désinences ne doivent pas donner moins de mal à ceux qui les lisent qu'à ceux qui les entendent» (Balzac Comédie vi 645). La modernité des procédés de Balzac a de quoi surprendre quelquefois. Car le romancier saute à cet endroit d'un niveau de texte à un autre niveau. Ceux qui « entendent » les paroles de Nucingen, ce sont les personnages, les interlocuteurs du baron dans les dialogues. J'ai déjà dit qu'en fait ils le comprenaient fort bien (à l'exception peut-être de César Birotteau, qui a l'esprit obscurci par son honnêteté, ou 
par autre chose encore, comme on va voir). Ceux qui les «lisent »: mais c'est nous, les lecteurs. Ceci va bien plus loin qu'une simple adresse au lecteur, qui est monnaie courante dans le roman au XIX ${ }^{\text {ème }}$ siècle. Au détour de ces quelques mots, Balzac oublie l'histoire qu'il raconte et se place au niveau de l'écriture, dont la lecture est l'autre face. C'est comme s'il nous confiait, presque involontairement, un secret d'écriture : moi, le romancier, j'ai écrit (transcrit, inscrit) l'accent de mon personnage de telle sorte que les discours qu'il tient soient un casse-tête pour le lecteur. Cette hypothèse est corroborée par les traces conservées du travail de Balzac. Voici un passage de La Maison Nucingen, avec la note de l'éditeur du texte, Pierre Citron. Il est à noter que nous avons là la toute première réplique attribuée au baron dans $L a$ Maison Nucingen, il s'agit donc d'un texte inaugural :

"Hé pien! ma ponne amì , dit Nucingen à du Tillet en tournant le boulevard, location est pelle bire ebiser Malfina: fous serez le brodecdir teu zette baufre vamile han plires, visse aurez eine vamile, ine indérière; fous drouferez eine mison doute mondée, et Malfina cerdes esd eine frai dressor. » (Balzac Comédie vi 358)

La note de l'éditeur dans la Pléiade concerne la variante $\mathrm{h}$ :

h. ma ponne ami, épr. 7: mon ami, ant. La transcription phonétique du jargon de Nucingen, sauf exceptions qui seront indiquées, a été introduite par Balzac, en plusieurs fois, de la $6^{e}$ à la $8^{e}$ épreuve. Le détail de ces transformations est trop complexe pour être reproduit intégralement (Balzac Comédie vi 1278).

On voit que c'est tout subitement, sur épreuves, précisément sur les épreuves $n^{\text {os }} 6,7$ et 8 - placé donc devant des pages imprimées de son livre, des pages qui s'éloignent déjà du travail de l'invention romanesque, des pages qui confrontent l'écrivain à un texte inscrit, gravé, objectivé, en voie de lui échapper - , c'est là que l'écrivain a été comme saisi de frénésie et qu'il a entrepris, de façon presque compulsive, de perfectionner sa transcription de «l'accent». Cet accent n'était pas alors une invention absolue, il en avait doté Nucingen dès Le Père Goriot, mais dans ce roman le banquier apparaissait à peine et ne prononçait que quelques mots. Dans La Maison Nucingen Balzac se met à ce travail avec une telle minutie que Pierre Citron baisse les bras : «le détail de ces transformations est trop complexe pour être reproduit intégralement ». «Transcription phonétique »? Je crois que l'éditeur de la Pléiade se trompe. Il s'agit de véritables créations. Quant à la frénésie de Balzac, elle ressemble à une ivresse : voyez l'image du «baume exhilarant » dans César Birotteau. Le parfumeur est comme prédestiné à être drogué par la parole du baron, laquelle à son tour, à un autre niveau, agit comme une drogue sur son créateur. Plus exactement, c'est le travail sur le langage de Nucingen qui est la vraie drogue.

«Paroles semi-françaises », dit le narrateur dans César Birotteau (Balzac Comédie vi 232). Il fallait donc un intrus, un étranger au sens fort, pour bousculer aussi radicalement la langue qui était familière à l'écrivain, il fallait cette sorte de alien tombé on ne sait d'où, le Juif-polonais-alsacien-allemand qui règne simultanément 
dans la sphère des mystérieux flux financiers, pour libérer l'écriture de Balzac et lui ouvrir la possibilité d'inventer une langue inconnue et dangereuse ${ }^{8}$.

Dans la création de mots monstrueux, Balzac va beaucoup plus loin que Victor Hugo. Le code des transformations vole en éclats. Une fois la maison devient mison, une autre fois ce sera mésson. Il n'y a plus de loi. Ce n'est plus un savant dosage de sourdes et de sonores, c'est une folie. Des mots français parfaitement identifiables surgissent, parfaitement énigmatiques dans le contexte: des mots comme location, pelle, frai, fous, chai, chausse. Des mots qui flottent et nous entraînent vers de vagues dérives, un peu comme dans l'aquarium de Jules Laforgue. On pense aux rébus, ou encore aux journaux froissés : on se souvient de ces comparaisons utilisées par Freud pour expliquer le travail du rêve. Inversement, tout un lexique de la vie quotidienne s'est englué, s'est littéralement englouti dans la pâte du baragouin de Nucingen perfectionné par Balzac. Des mots tels que épouser, protecteur, en pleurs, un intérieur, un trésor, je soupire, quelque chose d'inconnu, en voiture à minuit au bois de Vincennes émergent à grand peine du magma, on les repêche quand on se donne une peine infinie. On peut penser que Balzac n'expérimente pas seulement sur les mots, il camoufle aussi, avec une sorte de malignité et jusqu'à l'extrême limite de la possibilité de reconnaissance, les choses que les mots désignent. Il met ainsi en question à la fois la représentation du monde et la représentation de la langue.

Si La Comédie humaine est bien l'espace où se déploie la capacité de créer de l'écrivain-démiurge, le baragouin de Nucingen est comme l'acide qui ronge ce monde de mots de l'intérieur, qui en fait mesurer - peut-être même désirer - la fragilité. Par l'intermédiaire de Nucingen, l'artiste expérimente le vertige de son néant. Je pense à Rimbaud, à son «atroce scepticisme». En écrivant le poème «Vies », Rimbaud pensait peut-être à Balzac. Ne dit-il pas : «j'ai illustré la comédie humaine »? Balzac de son côté le rejoint dans une expérience extrême. Peut-être pourrait-il dire : «Je suis réellement d'outre-tombe »?

\footnotetext{
${ }^{8} \mathrm{~N}$ 'importe quel Alsacien ne ferait pas l'affaire : le personnage de Kolb, dans Illusions perdues, est paradoxalement fidèle à la langue française tout en la massacrant selon le code des transformations conventionnel : «[...] quoique chaie l'air d'ein Hallemante [...] che suis ein frai Vrançais » (Balzac Comédie v 621).
} 


\section{Bibliographie}

- Balzac, Honoré de. Illusions perdues in La Comédie humaine. Paris: Gallimard, Bibliothèque de la Pléiade, t. v, 1977

- Balzac, Honoré de. La Maison Nucingen, César Birotteau, Splendeurs et misères des courtisanes in La Comédie humaine. Paris : Gallimard, Bibliothèque de la Pléiade, t. vi, 1977

- Goncourt, Edmond et Jules. Journal. Paris : Robert Laffont, «coll. Bouquins », t. i, 1989

- Hugo, Victor. Le Rhin in CEuvres complètes. Voyages. Paris : Robert Laffont, « coll. Bouquins », 2002

- Hugo, Victor. Écrits politiques. Paris : Le Livre de Poche, 2001

- Huré, Jacques. "Allemagne et Orient», Cahiers Gérard de Nerval, n spécial Les affinités germaniques de Nerval. Bourg en Bresse: Cahiers de la Société Gérard de Nerval, 1984

- Jackson, E. John. Passions du sujet. Essais sur les rapports entre psychanalyse et littérature. Paris : Mercure de France, 1990

- Nerval, Gérard de. Lorely in CEuvres complètes. Paris : Gallimard, Bibliothèque de la Pléiade, t. iii, 1993

- Savy, Nicole. Victor Hugo, voyageur de l'Europe. Bruxelles : Éditions Labor, 1997

- Terrasse-Riou, Florence. Balzac. Le roman de la communication. Paris : SEDES, 2000 